

EDITORIAL Methodo 2019;4(3):69-70

https://doi.org/10.22529/me.2019.4(3)01

Solicitado 01 Jul. 2019 | Recibido 03 Ago. 2019 | 20 Ago. Aceptado 2019 |

Publicado 30 Sep.2019

\title{
Entornos virtuales de aprendizaje en ciencias de la salud
}

\section{Virtual learning environments in health sciences}

La actualidad está marcada por el desarrollo cada vez más vertiginoso de la tecnología. Este hecho incide necesariamente en el proceso formativo de nuestros estudiantes

Los EVA (entornos virtuales del aprendizaje) son herramientas para facilitar la transmisión del conocimiento mediado por la informática

Las posibilidades de los EVA aplicados al aprendizaje en estudiantes universitarios permiten complementar la enseñanza tradicional a través de los distintos dispositivos, optimizando la utilización de los recursos disponibles.

Para que estos entornos sean atractivos para el estudiante, es necesario modificar los modelos convencionales de aprendizaje buscando métodos pedagógicos centrados en el alumno reservando el rol del docente como un acompañante, guía, facilitador y, principalmente, creador de oportunidades de aprendizaje.

La sugerencia principal de los investigadores, es que en lugar de usar la tecnología para difundir la información, incluyendo el uso de plataformas gestoras de aprendizajes o LMS (como el Moodle) como solo un medio para la presentación de los contenidos, los EVA deben ser utilizados como herramientas para involucrar a los estudiantes en el pensamiento crítico reflexivo acerca de las ideas que están estudiando, estimular el trabajo colaborativo y el ABP o aprendizaje basado en problemas ${ }^{1}$.

Para lograr esto es fundamental que el entorno virtual de aprendizaje provea al alumno de una interacción directa y fluida con sus pares y docentes, esta interacción es favorecida a partir de la creación de situaciones de "resolución de problemas, en el desarrollo de proyectos, en la creación conjunta de productos, etc., mediante la comunicación y la discusión con el docente y los demás compañeros"2.

Además de las ventajas ya conocidas de los EVA en la enseñanza, la sociedad de hoy demanda profesionales de la salud cada vez mejor formados en nuevas tecnologías para asegurar una óptima integración en el ámbito, pero también para que esté familiarizado con los procesos y así generar mejores posibilidades en la formación continua de nuestros egresados, ya que existe una tendencia cada vez mas instalada de la capacitación a distancia en formación de postgrado debido a la cada vez mayor necesidad de actualización y la necesidad de combinar educación y trabajo.

Revista Methodo: Investigación Aplicada a las Ciencias Biológicas. Facultad de Medicina. Universidad Católica de Córdoba. Jacinto Ríos 571 Bo Gral. Paz. X5004FXS. Córdoba. Argentina. Tel.: (54) 3514517299 
Un punto ineludible en este desarrollo es el compromiso institucional, estos procesos necesitan el apoyo constante a los docentes, ya sea en formación, soporte y reconocimiento, no solo a nivel de las universidades sino desde organismos gubernamentales.

La gran mayoría de los trabajos acuerdan en señalar el nivel de conformidad de los alumnos en la utilización de estas plataformas, dato esperado ya que facilitan el acceso a la información y el aprendizaje respetando tiempos individuales, como contrapartida encontramos que no en todos los casos se ven respuestas positivas en la evaluación de la incorporación del conocimiento posterior a su uso, esto queda directamente relacionado al compromiso, conocimiento y constancia en el diseño y seguimiento del recurso, como toda herramienta pedagógica.

El futuro está enmarcado con diferentes desafíos, uno de ellos sigue siendo el recurso docente, otro a mi entender es saber escuchar al alumno, me refiero a comprender el porqué de la respuesta dispar en la utilización y valoración de los EVA.

También tenemos que ser conscientes que somos solo parte de la evolución de las metodologías de enseñanza no presénciales, ya que desde hace mucho tiempo comenzando con la educación por correspondencia y atravesado luego por la multimedia y la informática, esta transformación metodológica en la educación muestra un progreso vertiginoso, lo nos asegura nuevas y buenas noticias. La inteligencia artificial y el diseño de pacientes virtuales ya es hoy una realidad en aulas universitarias.

Por todo esto creo que hoy, la discusión ya no pasa por decidir si necesitamos incluir o no los entornos digitales en la enseñanza de las ciencias de la salud, eso ya nadie lo duda, ahora tenemos que ser capaces de aprovechar la cada vez mejor oferta tecnológica y optimizar su uso ya no para facilitar nuestra enseñanza si no el aprendizaje de nuestros alumnos.



Dr. Sergio Ruffino

Secretario de Grado

Facultad de Ciencias de la Salud.

\footnotetext{
${ }^{1}$ Jonassen DH, Carr C, Yueh HP. Computers as Mindtools for Engaging Learners in Critical Thinking. Tech Trens 1998; 43 (2):24-32.

${ }^{2}$ Gros Salvat B (ed.) Evolición y retos de la educación virtual. Construyendo el e-learning del siglo XXI.

Primera edición, editorial UOC, Barcelona, España. 2011.
} 